\title{
ISSUES IN ACCELERATION OF A MUON BEAM FOR A NEUTRINO FACTORY*
}

\author{
J. Delayen, D. Douglas, L. Harwood, V. Lebedev ${ }^{\dagger}$, C. Leemann, and L. Merminga, \\ TJNAF, Newport News, VA, 23606 USA
}

\begin{abstract}
We have developed a concept for acceleration of a large phase-space, pulsed muon beam from $190 \mathrm{MeV}$ to 50 $\mathrm{GeV}$ as part of a collaborative study of the feasibility of a neutrino factory based on in-flight decay of muons. The muon beam's initial energy spread was $\sim 20 \%$ and each bunch had the physical size of a soccer ball. Production of the muons will be quite expensive, so prevention of loss due to scraping or decay is critical. The former drives the system to large apertures and the latter calls for high realestate-averaged gradients. The solution to be presented utilizes a $3 \mathrm{GeV}$ linac to capture the beam, a 4-pass recirculating linac to get the beam to $10 \mathrm{GeV}$, and then a 5-pass linac to get the beam to $50 \mathrm{GeV}$. Throughout the system, longitudinal dynamics issues far outweighed transverse dynamics issues. This paper focuses on the issues surrounding the choice of superconducting rf structures over copper structures.
\end{abstract}

\section{INTRODUCTION}

A beam of relativistic muons can conceivably be used to produce a controlled, directed beam of neutrinos as the muons decay [1]. A study of this idea was initiated by the Muon Collider Collaboration and was carried out under the stewardship of FermiLab. The concept was based on: 1) production of the muons with a proton beam, 2) a muon cooler, 3) a muon acceleration section, and 4) a muon storage ring with long straight sections in which most of the muons decay and form a collimated neutrino beam.

JLab was asked to contribute to the study by conceptualizing the acceleration of a low-momentum, large phase-space muon beam to relativistic energy. The beam specifications are listed in Table 1 . The initial beam properties are determined by the capability of the muon cooling section. The final beam parameters are driven by the acceptance of the storage ring in which the muons decay into neutrinos.

In addition to achieving the desired final beam properties, the muon accelerator must preserve as many of the expensive-to-produce muons as possible for delivery to the storage ring. This requires achieving a high "real estate gradient", i.e. MV/m of total path length. Finally, a cost-effective system is highly desirable.

It was determined that a three-stage acceleration scheme would be needed. The first stage is a capture

*This work was supported by the U.S. Department of Energy Contract NumberDE-AC05-84-ER40150

${ }^{\dagger}$ Present address: FermiLab, Batavia, Ill 60510 USA linac that gets the muons to relativistic energies as rapidly as possible so as to minimize the decay losses and also to damp the emittance to a manageable size; the energy gain of this linac was set at $3 \mathrm{GV}$. Next came two cascaded recirculating linacs. Recirculating linacs (RLA's) were chosen in order to minimize the contribution of the relatively expensive accelerating structures and supporting $\mathrm{rf}$ systems. For a zero-emittance beam, the number of recirculations is arbitrary. However, for a beam with this very large emittance, beta-function control is critical and, since beams with difference energy experience the same fields in the linacs' focussing elements, the ratio of input-to-output energies must be constrained. Thus, four passes was felt to be the maximum that could be used. This pre-accelerator RLA was used to bring the muons from $3 \mathrm{GeV}$ to $10 \mathrm{GeV}$. The final $40 \mathrm{GeV}$ of acceleration was provided by a second RLA which uses five passes.

The next major step was to examine the details of the accelerating structures. The extraordinarily large initial phase space provided a particular challenge. How this problem was addressed follows.

Table 1. Muon beam properties

\begin{tabular}{|c|c|}
\hline Parameter & Value \\
\hline Initial energy & $190 \mathrm{MeV}$ \\
\hline Final energy & $50 \mathrm{GeV}$ \\
\hline Initial transverse emittance & $\varepsilon_{\mathrm{x}, \mathrm{y}}=1.5 \mathrm{~mm}-\mathrm{rad}$ \\
\hline Initial longitudinal emittance & $\sigma_{\mathrm{z}} \times \sigma_{\mathrm{E}}=12 \mathrm{~cm} \mathrm{x} \mathrm{11 \%}$ \\
\hline $\begin{array}{c}\text { Micro-pulse spacing } \\
\text { (frequency) }\end{array}$ & $5 \mathrm{nsec}(200 \mathrm{MHz})$ \\
\hline $\mathrm{N}_{\text {micro-pulses }}$ per pulse & 30 \\
\hline $\mathrm{N}_{\text {micro-pulses per macro-pulse }}$ & $120(=30 \times 4)$ \\
\hline Macro-pulse length & $\begin{array}{c}\text { four } 150 \mathrm{nsec} \text { pulses } \\
\text { with } 250 \mathrm{nsec} \text { pulse- } \\
\text { to-pulse separation }\end{array}$ \\
\hline $\mathrm{N}_{\mu}$ per macro-pulse & $3 \mathrm{x} 10^{12}$ \\
\hline Macro-pulse repetition rate & $15 \mathrm{~Hz}$ \\
\hline Final energy & $50 \mathrm{GeV}$ \\
\hline Final emittance (normalized) & $3 \mathrm{~mm}-\mathrm{mrad}$ \\
\hline Final energy spread & $\pm 2 \%$ \\
\hline
\end{tabular}

\section{FREQUENCY CHOICE}

Choices for the rf frequency were quite limited. First, the bunch spacing of $5 \mathrm{nsec}$ dictated the use of $200 \mathrm{MHz}$ or one of its harmonics. Higher frequencies were desirable because the structures are smaller which leads to them being cheaper and requiring less rf power in $\mathrm{CU}$ 
structures because of reduced wall losses and less rf power in SRF structures because of reduced need for power to control microphonics effects (thereby making the rf system less expensive, as well).

Conversely, the large emittance drove the study toward lower frequencies. Assuming an optimistic transverse $\beta$-function of $3 \mathrm{~m}$, the beam would have a $1 \sigma$ radius of $7 \mathrm{~cm}$. Typically, one wants to have a "clear aperture" of at least $3 \sigma$; so the structures would need an inner diameter of $>20 \mathrm{~cm}$. Lowering the frequency yields structures with larger apertures as a natural result.

Simultaneously, the longitudinal emittance sets a limit on the frequency. The input beam rms bunch-length is $12 \mathrm{~cm}$; this represents $8 \%$ of the wavelength at $200 \mathrm{MHz}$ or $29^{\circ}$. If the pulse is riding the crest of the rf wave, the $29^{\circ}$ length yields a $\pm 1.6 \%$ energy spread, which is comparable to the desired energy spread at $50 \mathrm{GeV}$. If we double the nominal emittance in order to set the limit for transmission, as was done for the transverse emittance, we get an energy spread of $\pm 6 \%$ which is well above the final goal. If $400 \mathrm{MHz}$ were to be used, then the core of the beam would have an energy spread of $\pm 6 \%$ and the full transmission number would be $\pm 23 \%$. Clearly, the values at $400 \mathrm{MHz}$ are untenable.

Consideration was given to the effects of adiabatic damping. Any damping would potentially be counterbalanced by the fact that, at least in the capture linac, the beam would be running off-crest which would further increase the energy spread in absolute terms. A brief study showed that the adiabatic damping would help the $200 \mathrm{MHz}$ case but would not solve the problem at $400 \mathrm{MHz}$ and permit its use in either the capture linac or in the pre-accelerator RLA.

Manipulation of the longitudinal phase space was also considered as a way to mitigate the problems. However, it was found that the transverse and longitudinal phase spaces tended to interact strongly, as might be expected with emittances as large as we have in this situation. Rotating the bunch-length into energy spread simply increased the chromatic effects of the focussing elements and thereby increased the difficulty of realizing the $1 \mathrm{~m}$ beta-functions mentioned previously. Conversely, rotating the energy spread into bunch length helped the transverse focussing but increased the net energy spread and additionally increased the distortion of the longitudinal phase space.

It was felt that the only viable solution for the capture linac and pre-accelerator RLA was to use the lowest possible rf frequency. Thus, $200 \mathrm{MHz}$ was selected as the rf frequency for the capture linac and the pre-accelerator linacs. There is ongoing evaluation of the viability of using $400 \mathrm{MHz}$ in the primary RLA, i.e. the one that accelerates the beam from $10 \mathrm{GeV}$ to $50 \mathrm{GeV}$.

\section{RF POWER OPTIONS}

Several natural time scales are present in the beam structure. Each micro-pulse is $0.4 \mathrm{nsec}$ long. Thirty micro-pulses form a $150 \mathrm{nsec}$ long pulse. Four pulses form a $1.4 \mu$ sec long macro-pulse. The macro-pulses occur at $15 \mathrm{~Hz}$. The average current varies enormously over these time scales, thus the average power needed for beam acceleration varies.

A calculation of the decay rate of the muons showed that an average gradient of $5 \mathrm{MV} / \mathrm{m}$ would be needed to achieve an acceptable survival rate. It was estimated that the gradient in a cavity would need to be $\sim 15 \mathrm{MV} / \mathrm{m}$ in order to achieve $5 \mathrm{MV} / \mathrm{m}$ as the "real estate averaged" gradient. As a single cell of an elliptical $200 \mathrm{MHz}$ cavity is $\sim 0.75 \mathrm{~m}$ long, the voltage gain of the cell is then 11.25 MV. The resulting power levels for the previously mentioned time scales are shown in Table 2. It should be noted that these values are for a single cell.

Table 2. Beam Power Delivered by a Single Cell

\begin{tabular}{|c|c|c|}
\hline & $\underline{\text { Time }}$ & $\underline{\text { Power }}$ \\
\hline Micro-pulse & $0.4 \mathrm{nsec}$ & $112 \mathrm{MW}$ \\
\hline Pulse & $150 \mathrm{nsec}$ & $9 \mathrm{MW}$ \\
\hline Macro-pulse & $1.4 \mu \mathrm{sec}$ & $4 \mathrm{MW}$ \\
\hline
\end{tabular}

Klystrons of this power do not presently exist; however, it is conceivable that such could be developed, at least at the $10 \mathrm{MW}$ level. Even if a single klystron proved insufficient, power combining schemes exist. However, these values exceed the state of the art in coupler technology, which is $5 \mathrm{MW}$ in this frequency range.

Since it is likely impossible to provide the $112 \mathrm{MW} /$ cell needed to directly drive the beam, we need to see what happens to the voltage during the $150 \mathrm{nsec}$ pulse or $1.4 \mu \mathrm{sec}$ macro-pulse. An elliptically shaped cell would have a stored energy of $\sim 1 \mathrm{~kJ}$ if the accelerating field is $15 \mathrm{MV} / \mathrm{m}$. Table 3 lists the energy imparted to the beam by a single cell at $15 \mathrm{MV} / \mathrm{m}$. It also lists the effect on the field if there is no rf power input to the cavity; this provides a worst-case estimate of what happens to the beam's energy spread independent of the capabilities of the klystron and coupler. In none of the three cases does the field droop constitute a significant fraction of the input energy spread or of the desired final energy spread.

\begin{tabular}{|c|c|c|}
\hline & Energy gain & $\frac{\text { Field droop }}{\text { with no rf }}$ \\
\hline Micro-pulse & $0.05 \mathrm{~J}$ & $<0.01 \%$ \\
\hline Pulse & $1.4 \mathrm{~J}$ & $0.07 \%$ \\
\hline Macro-pulse & $5.4 \mathrm{~J}$ & $0.27 \%$ \\
\hline
\end{tabular}

The cell's $1 \mathrm{~kJ}$ of stored energy is a major reason for the minimal field droop as the beam extracts energy. There is a negative aspect to this large energy content, however. The cell must be filled each time the system is pulsed. If the system were to be pulsed on the micro-second time scale of the macro-pulse's length, then giga-watt scale rf power would have to be produced and delivered to the cell. As stated earlier, this is well beyond the state-of-theart. If one stays within the mega-watt scale mentioned 
earlier, one is driven to a fill-time of order a millisecond $(=1 \mathrm{~kJ} / 1 \mathrm{MW})$.

\section{SRF VS WARM RF}

In the previous section we found that the present coupler technology restricts the duration of the rf pulse to be of order 1 msec. With pulses of this length, resistive losses in the walls of a $\mathrm{Cu}$ cavity could be an issue. At $200 \mathrm{MHz}$, an intrinsic Q of $\sim 10^{5}$ might be expected for a $\mathrm{Cu}$ cavity. This leads to a power dissipation of $\sim 12 \mathrm{MW}$ per cell. So, in order to simply sustain the field in the cavity it would be necessary to generate and deliver $\sim 12 \mathrm{MW}$. If an assumption is made that one could deliver $5 \mathrm{MW}$ to each cell, it would be possible to sustain a field of $10 \mathrm{MV} / \mathrm{m}$. This leads to a "real estate averaged" gradient of $\sim 3.5 \mathrm{MV} / \mathrm{m}$, which would lead to excessive muon losses due to in-flight decay. Alternatively, if one computes the total amount of required rf power for $15 \mathrm{MV} / \mathrm{m}$ operation, one sees that one would need to install $5 \mathrm{GW}$ of $\mathrm{rf}$ power to feed the 445 cells of the capture linac and RLA1, i.e. neglecting RLA2. If one assumes that it is possible to use $400 \mathrm{MHz}$ for RLA2, it's 1386 cells would require $\sim 2 \mathrm{GW}$ for resistive losses. We are thus looking at $7 \mathrm{GW}$ of rf simply to drive the resistive losses. This is not an appealing prospect. Thus, if $\mathrm{Cu}$ cavities are used we are left with the choice between excessive loss of the muons and the need to install an enormous amount of rf power.

SRF provides a possible alternative. $200 \mathrm{MHz}$ cavities are terra incognita, however, so performance is uncertain. Present technology achieves $\mathrm{Q}_{0}>10^{10}$ at $15 \mathrm{MV} / \mathrm{m}$ at $1.5 \mathrm{GHz}$ at $2 \mathrm{~K}$. $\mathrm{Q}_{0}$ generally increases with decreasing frequency. The cryogenics system cost would be less if the system were operated at $4.5 \mathrm{~K} ; \mathrm{Q}_{0}$ decreases as the temperature increases because of the BCS resistance. $\mathrm{Q}_{0}=6 \times 10^{9}$ was assumed to be a reasonable estimate of $\mathrm{Q}_{0}$ at $200 \mathrm{MHz}$ and $4.5 \mathrm{~K}$. This leads to an instantaneous dissipation of 200(25) W/cell for the 200(400) MHz cells. For the entire accelerator, the instantaneous dissipation would be $\sim 124 \mathrm{~kW}$. It would not be necessary to supply this level of cryogenic cooling, however. One could utilize the heat capacity of the cavity material and of the helium bath to sustain the conditions during a macro pulse. Assuming the $\mathrm{rf}$ is "on" for $\sim 1 \mathrm{msec}$, the timeaveraged dissipation is only $1.9 \mathrm{~kW}$ and would be at $4.5 \mathrm{~K}$, which is far less than the present capacity of the Jefferson Lab cryogenics plant.

The dynamic losses are not the totality of the cryogenic load; static losses must be added. An estimate of the total static load is typically done by estimating the static load per cryomodule and then multiplying by the number of cryomodules. For this study it was assumed that the majority of the cavities of the capture linac and RLA1 would be arranged in 57 cryomodules with eight cells per cryomodule. RLA2 would have 87 cryomodules each with sixteen of the $400 \mathrm{MHz}$ cells. An exact value of the static load requires a detailed design of a cryomodule; such a detailed design was not executed. Instead it was assumed that the static losses for each cryomodule would be comparable to those of one of the similarly-size CEBAF cryomodules, i.e. $16 \mathrm{~W}$. Thus, the 144 cryomodules, each at $16 \mathrm{~W}$ per cryomodule, would have $2.3 \mathrm{~kW}$ of static losses. Thus, the total cryogenic load would be $4.2 \mathrm{~kW}$, which is not an extraordinarily large load. For example, the $4.5 \mathrm{~K}$ capacity of the present JLab cryogenics plant is $10 \mathrm{~kW}$.

The primary driver for the $\mathrm{rf}$ power requirement in an srf option is dealing with microphonic detuning of the cavity. In the limit that the detuning bandwidth is much larger than the intrinsic bandwidth, the power required for control is given by

$$
\mathrm{P}_{\text {control }} \cong 2 \pi(\delta \mathrm{F}) \mathrm{U}
$$

where $\delta \mathrm{F}=$ detuning bandwidth and $\mathrm{U}=$ stored energy. Estimating the detuning bandwidth for the $200 \mathrm{MHz}$ cavities is rather speculative. The value used for planning the JLab $12 \mathrm{GeV}$ project is $25 \mathrm{~Hz}$; that system benefits from being a cw system which thereby does not have a low-frequency mechanical driver, i.e. the $15 \mathrm{~Hz}$ Lorentz forces. The SNS project, which is an SRF linac that is pulsed at $60 \mathrm{~Hz}$, is using $100 \mathrm{~Hz}$ for it's planning. For the present estimate, we have used $80 \mathrm{~Hz}$, which is somewhat reduced from the SNS value because of the substantially shorter pulse in the present study. As stated before, the stored energy at $15 \mathrm{MV} / \mathrm{m}$ is $1 \mathrm{~kJ}$. This then yields a control power of $502 \mathrm{~kW}$. This is gratifyingly within the state-of-the-art for coupler technology. Using this value for the $200 \mathrm{MHz}$ cells and $125.6(=502 / 4) \mathrm{kW}$ for the $400 \mathrm{MHz}$ cells, we see that the total rf power is $0.45 \mathrm{GW}$. This compares favorably to the $10 \mathrm{GW}$ needed to sustain the wall losses of the Cu option.

\section{CONCLUSION}

A choice of $200 \mathrm{MHz}$ is forced by the large emittance of the incoming muon beam and the desire to have a small energy spread at $50 \mathrm{GeV}$. Coupler technology limits the power/cell to <10 MW; consequently the cavity filling time must be of order $1 \mathrm{msec}$, which is substantially longer than any of the natural time-frames of the pulse structure. Fortunately the $1 \mathrm{~kJ}$ of stored energy in a $200 \mathrm{MHz}$ cavity at $15 \mathrm{MV} / \mathrm{m}$ (which is needed for an acceptable survival rate of the muons) results in a tolerable voltage droop during the macro-pulse. The $15 \mathrm{MV} / \mathrm{m}$ field requirement also results in an intolerably expensive resistive loss if $\mathrm{Cu}$ cavities are used. Use of SRF cavities would result in modest cryogenics loads and rf power requirements. Use of SRF cavities seems to be the only way to cost effectively accelerate the muons to $50 \mathrm{GeV}$ in the context of this study.

At this time the principal unknown is the SRF performance of a $200 \mathrm{MHz}$ cavity. R\&D on this topic would be beneficial to further progress.

\section{REFERENCES}

[1] http://www.fnal.gov/projects/muon_collider/nu/study/r eport/machine_report 